\title{
Species of Uromyces (Pucciniales, Basidiomycota) on Loranthaceae
}

\author{
Omar Perdomo Sánchez ${ }^{1}$ \& Meike Piepenbring²
}

${ }^{1}$ Dominican Society of Mycology, P. O. Box 151, 10101 Santo Domingo, Dominican Republic; ${ }^{2}$ Institute for Ecology and Evolution, Cluster for Integrative Fungal Research (IPF), Goethe University, Max-von-Laue-Str. 13, 60438 Frankfurt am Main, Germany

Author for correspondence: Omar Perdomo Sánchez, e-mail: omarpaino@gmail.com

\begin{abstract}
Two new species of Uromyces with reticulated teliospores are compared with nine species of this genus known from Loranthaceae. The new species Uromyces bahiensis from Brazil has smaller spores than all known species of Uromyces with reticulate teliospores on Loranthaceae. Uromyces struthanthi from Panama is characterized by long teliospore pedicels and spinose-echinulate aecidiospores. In addition, new details of ornamentation of aecidiospores and teliospores of known species are presented.
\end{abstract}

Key words: Uromyces, Loranthaceae, new species, Pucciniales.

\section{INTRODUCTION}

Pucciniales are the largest order of plant parasitic fungi in the Basidiomycota, including about 9000 species. Nevertheless, numerous unknown species are supposed to exist, especially in the tropics (Shivas \& Hyde, 1997). The genus Puccinia has the greatest number of species and Uromyces is the second largest genus of rust fungi. It includes more than 600 species and despite its high relevance it has not yet been monographed. Uromyces spp. differ morphologically from Puccinia spp. only by having 1-celled teliospores (Cummins \& Hiratsuka, 2003). This characteristic, however, has probably arisen independently several times in Pucciniaceae and alone is not sufficient for recognizing a monophyletic genus (van der Merwe et al., 2007). Uromyces species parasitise monocots and dicots throughout the world. More Uromyces species have been recorded from the Asteraceae, Euphorbiaceae, Fabaceae, Liliaceae, and Poaceae, than for other host families. Among the plant families that include hosts of Uromyces is the family Loranthaceae. It comprises more than 900 species of hemiparasitic shrubs. Most species occur in tropical and subtropical areas of Africa, America, Asia, and Europe, where they live on the stems or branches of their host plants (Vidal-Russell \& Nickrent, 2008). Nine species of Uromyces are known to have Loranthaceae as host, namely Uromyces circumscriptus Neger, Uromyces euphlebius Syd. \& P. Syd., Uromyces evastigatus Cummins, Uromyces loranthi H. S. Jacks., Uromyces nilagiricus T. S. Ramakr. \& K. Ramakr., Uromyces ornatipes Arthur, Uromyces phthirusae Mayor, Uromyces socius Arthur \& Holw., and Uromyces urbanianus Henn. Here this group of species is reexamined and two novel species of Uromyces occurring on this family are described.

\section{MATERIAL AND METHODS}

One specimen collected in the province of Chiriquí in Western Panama in 2003 was provided for this study by R. Kirschner and deposited at the Herbario Nacional of the Universidad de Panama (PMA) and at the Botanische Staatssammlung München (M) in Germany. The identification of the host plant for the new species is based on Kuiji (1978) and was compared with specimens available in the National Herbarium in Panama City (PMA). The other specimens cited below were loaned from the herbaria BPI and PUR. Uromyces nilagiricus was described from India, but no herbarium specimen could be located. Therefore, only the description published by Ramakrishnan \& Ramakrishnan (1950) was used.

Spores and handmade sections of sori were mounted in lactophenol and heated to boiling. They were examined with a Leitz Ortholux II light microscope (LM). Sizes in the species descriptions are based on at least 25 measurements of each structure. For scanning electron microscopy (SEM), air-dried material was mounted directly onto a specimen stub, sputtered with gold for $60 \mathrm{sec}$, and examined with a Hitachi S 4500 scanning electron microscope. Images were processed with Digital Image System 2.5. The terminology proposed by Cummins \& Hiratsuka (2003) was used to describe the life-cycle states.

\section{RESULTS AND DISCUSSION}

Ten species, including two new species of Uromyces on Loranthaceae are described and illustrated based on an investigation of herbarium material. The main morphological characteristics of these eleven species 
are summarized in Table1, including $U$. nilagiricus from India. The known distribution of most Uromyces species on Loranthaceae is restricted to Mexico, Central and South America, according to the data currently available. The Loranthaceae are assumed to have evolved on the Gondwana continent before the separation of South America and Africa (Vidal-Russel \& Nickrent, 2008). The specific geographical distribution of Uromyces species on Loranthaceae might, therefore, be explained by one or few jumps of rust fungi onto species of Loranthaceae from other host families after the separation of South America from Africa.

Uromyces bahiensis Perd.-Sánch., sp. nov. (Figures 1 A-D, 2A, 5 A-B, 6 1-3).

Holotype: BRAZIL, BAHIA: Vitória da Conquista. On living leaves of indet. Loranthaceae, 10 March 1984, J. F. \& M. M. Hennen 84-235 (HOLOTYPE: PUR 89046) [few I, III]. Brazil.

Etymology: Referring to the region of collection in

$$
\text { MycoBank \# MB805146. }
$$

Spermogonia not seen. Aecidia hypophyllous, 0.5-0.1 mm diam., subepidermal, erumpent, pulverulent, yellowish. Peridial cells (35-) 36-44 (-48) $\times(20-)$ 24-32 $(-38) \mu \mathrm{m}$, rhomboidal or angular, overlapping, outer wall smooth, inner wall verrucose, hyaline or slightly yellowish. Aecidiospores (30-) 32-38 (-40) × (21-) 28-33 (-35) $\mu \mathrm{m}$, catenulate, rhomboidal or angular, verrucose, hyaline or slightly yellowish. Uredinia not seen. Telia hypophyllous, subepidermal, in small yellowish-brown spots, $0.8-1 \mathrm{~mm}$ diam., grouped in areas of $2-2.5 \mathrm{~mm}$ diam., flattened to globoid, pulverulent, ruptured epidermis conspicuous. Teliospores 1-celled, oblong-ellipsoidal, (23-) 25-29 (-31) $\times(15-)$ 16-18 (-20) $\mu \mathrm{m}$, wall evenly $2 \mu \mathrm{m}$ thick, smooth, yellowish to brown, surface reticulate, germ pores not observed. Pedicels persistent, cylindrical, smooth, thinwalled, hyaline, short, 5-7 (-8) × 4-6 $\mu \mathrm{m}$. Basidia and basidiospores not observed.

On indet. Loranthaceae.

Distribution. Known only from Brazil.

Remarks. The new species $U$. bahiensis is morphologically close to $U$. circumscriptus in aecidiospore size. However, peridial cells are absent in aecidia of $U$. circumscriptus. The new species is characterized by having teliospores which are smaller than those of other known species of Uromyces on Loranthaceae which have reticulate teliospores.

Uromyces circumscriptus Neger, Anal. Univ. de Chile, 92: 328. 1895. (Figures 2 B, 5 C-D, 6 4-5).

Type. CHILE. VALDIVIA: 1895. On Struthanthus complexus Eichler, Neger n.n. [I, III] (PUR F2698).
Spermogonia not seen. Aecidia amphigenous, 0.5-1 $\mathrm{mm}$ diam., subepidermal, erumpent, pulverulent, grouped in areas of $1.5 \mathrm{~mm}$ diam., yellowish. Peridial cells not seen. Aecidiospores (28-) 31-36 (-42) × (25-) 27-31 (-32) $\mu \mathrm{m}$, catenulate, rhomboidal or angular, verrucose, hyaline to slightly yellowish. Uredinia not seen. Telia amphigenous, subepidermal, in small yellowish-brown spots, 0.8-1 $\mathrm{mm}$ diam., grouped in areas of 1-3 mm diam., pale brown, subpulverulent, ruptured epidermis conspicuous. Teliospores 1-celled, ellipsoidal, (32-) 36-41 (-43) × (17-) 19-23 $(-25) \mu \mathrm{m}$, lateral wall $2 \mu \mathrm{m}$ thick, distal part of the wall 4-7 $\mu \mathrm{m}$ thick, yellowish-cinnamon, rarely hyaline, surface reticulate-striate, germ pores not observed. Pedicels persistent, cylindrical, smooth, thin-walled, hyaline, short, $5(-7) \times 5 \mu \mathrm{m}$. Basidia and basidiospores not observed.

On Loranthus tetrandrus Ruiz \& Pavon, Loranthus sp., Phrygilanthus heterophyllus Tiegh, P. verticillatus (Ruiz \& Pav.) Eichler [= Loranthus verticillatus Ruiz \& Pavon], and Struthanthus complexus Eichler (Hennen et al., 1982, 2005; Lindquist, 1982; Mendes et al., 1998; Mujica \& Vergara, 1945; Mujica \& Oehrens, 1967).

Distribution. Known from Argentina, Brazil, and Chile (Hennen et al., 1982, 2005; Lindquist, 1982; Mendes et al., 1998; Mujica \& Vergara, 1945; Mujica \& Oehrens, 1967).

Additional specimens examined. ARGENTINA, MENDOZA: Tunuyán, Bella Vista. On Phrygilanthus verticillatus, 14 December 1946, Covas [I, III] (PUR F11721); MENDOZA, Las Heras, Quebrada del Potrero, 25 March 1956, A. Ruíz Leal 17.926 [I, III] (PUR F16897); BRAZIL: State unknown, city unknown. On Strhuthanthus complexus, 1 January 1896, Ule 2123 [III] (PUR N3910).

Remarks. Uromyces circumscriptus differs from $U$. urbanianus in the surface and size of teliospores and from $U$. evastigatus because teliospores are larger in U. evastigatus, and walls of aecidiospores of $U$. evastigatus are thicker at the apex (up to $12 \mu \mathrm{m}$ ). Furthermore, the ornamentation of teliospores of $U$. circumscriptus is reticulate-striate, while it is smooth to finely-densely verrucose in U. loranthi, reticulate in $U$. bahiensis, longitudinally striate in $U$. euphlebius, longitudinally rugose-striate in $U$. ornatipes, striate in $U$. phthirusae, longitudinally-striate in U. socius, verrucose-striate in $U$. urbanianus, and reticulate-foveate in U. struthanthi.

Uromyces euphlebius Syd., Ann. Mycol. 18: 154. 1920. (Figures 3A, 4A, 6 6-7).

Type. MEXICO, JALISCO: On Phoradendron sp., Reiche n.n. [II, III] (PUR 13782).

Spermogonia and Aecidia unknown. Uredinia amphigenous, subepidermal, in groups of 2-4 mm diam., cinnamon-brown, pulverulent, ruptured epidermis conspicuous. Urediniospores ellipsoidal to oblongellipsoidal, (42-) 44-49 (-52) × (21-) 22-25 (-26) $\mu \mathrm{m}$, wall golden-brown, sparsely echinulate, $2-2.5 \mu \mathrm{m}$ thick, germ pores 4 equatorial. Pedicels persistent or deciduous, 
Species of Uromyces (Pucciniales, Basidiomycota) on Loranthaceae

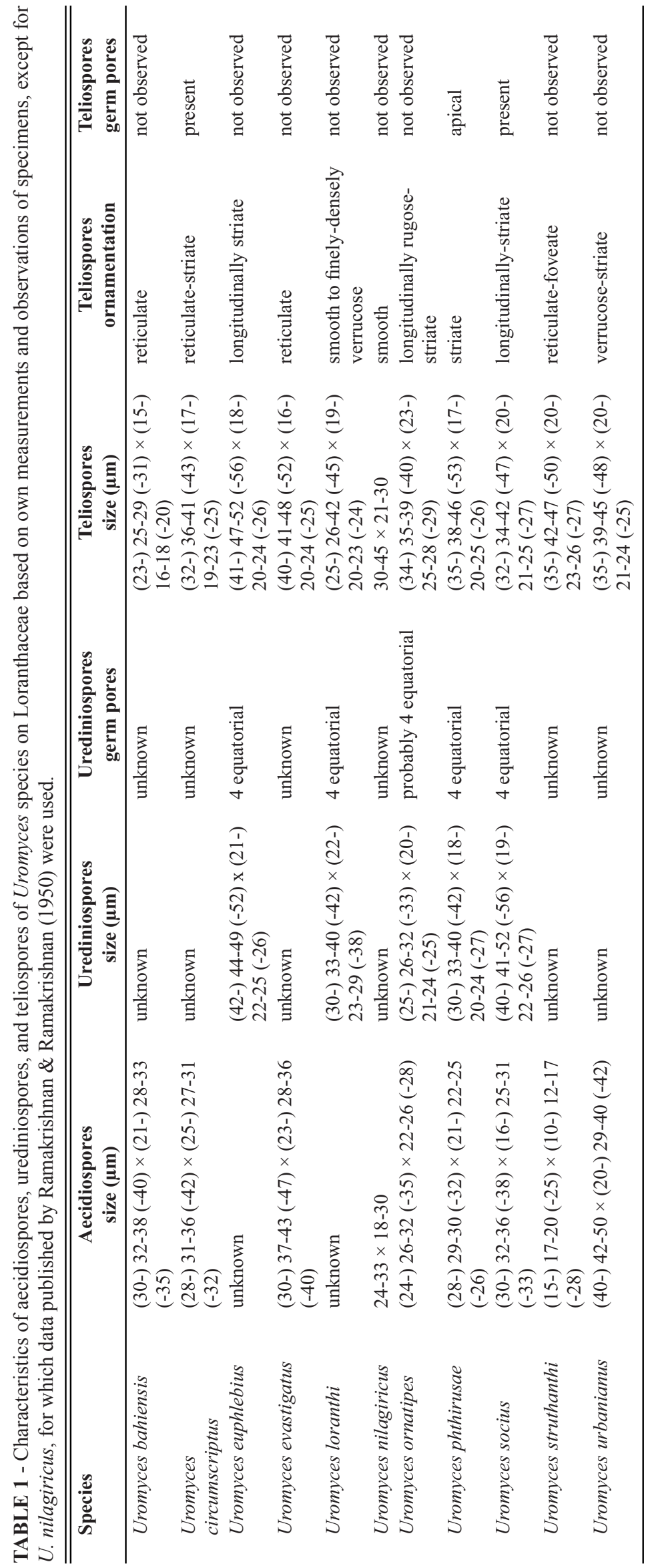




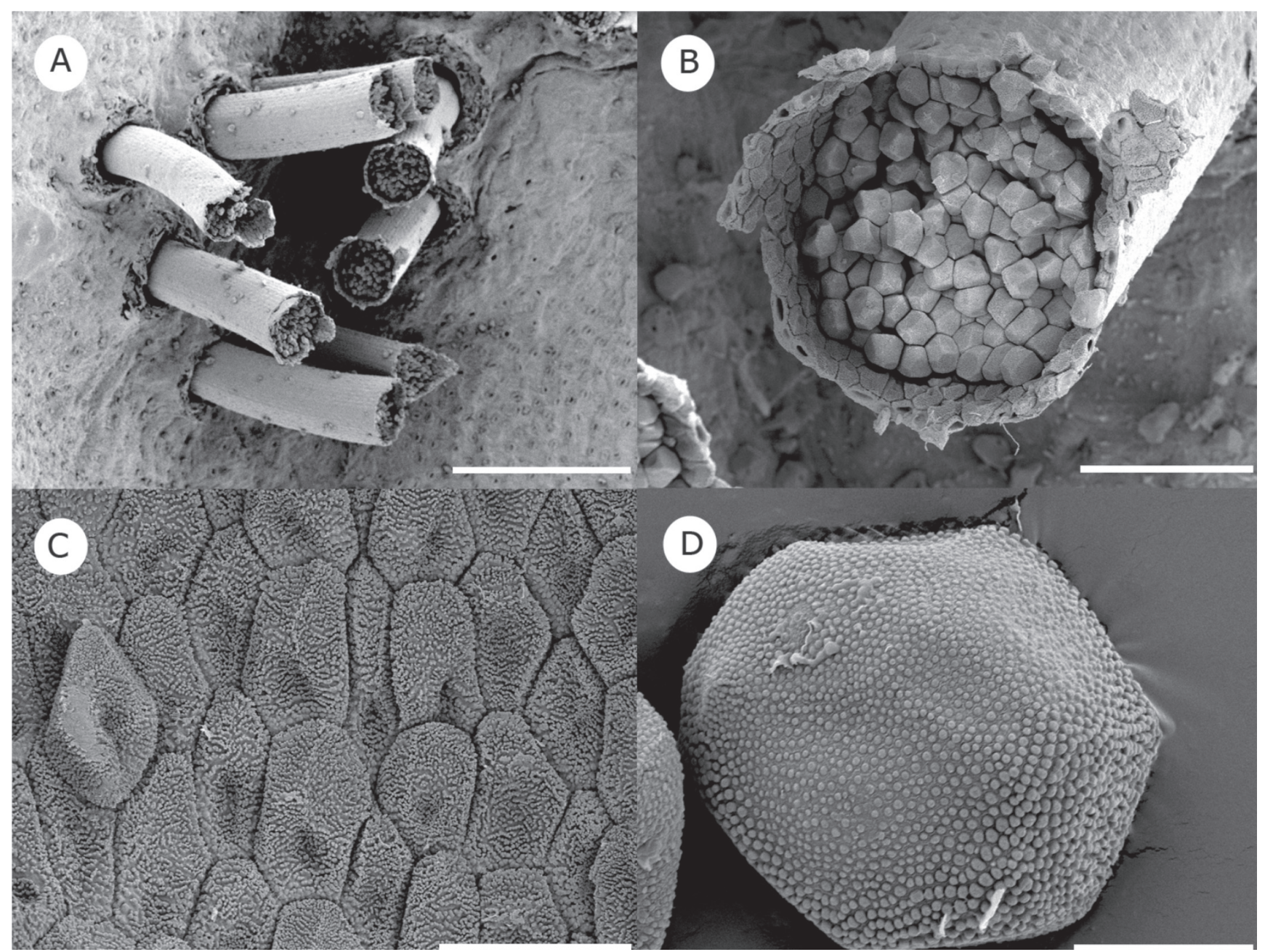

FIGURE 1 - Uromyces bahiensis, as seen under SEM. A. Aecidia (bar $=50 \mu \mathrm{m})$; B. Aecidium with peridial cells and aecidiospores (bar $=9 \mu \mathrm{m})$; C. Peridial cells as seen from the inner side of a peridium $(\mathrm{bar}=30 \mu \mathrm{m})$; D. Mature aecidiospore $(\mathrm{bar}=8 \mu \mathrm{m})$.

cylindrical, smooth, thin-walled, hyaline, short, 8-18 $(-20) \times 4-5 \mu \mathrm{m}$. Telia amphigenous, subepidermal, in small dark chocolate-brown spots, in 1-3 $\mathrm{mm}$ diam. groups, subpulverulent, ruptured epidermis conspicuous. Teliospores 1-celled, oblong, (41-) 47-52 (-56) × (18-) 20$24(-26) \mu \mathrm{m}$, lateral wall $2-2.5 \mu \mathrm{m}$ thick, distal wall 7-10 $\mu \mathrm{m}$ thick, chestnut-brown, longitudinally striate, germ pores not observed. Pedicels persistent, cylindrical, smooth, thin-walled, hyaline, 8-10 (-50) $\times$ 4-5 (-7) $\mu \mathrm{m}$. Basidia and basidiospores not observed.

Neotropical hosts. On Psittacanthus calyculatus (DC.) G. Don and Phoradendron sp. (Gallegos \& Cummins, 1981).

Known only for Mexico (Gallegos \& Cummins, 1981).

Additional specimen examined. MEXICO, SANTA MARIA: Cuernavaca, Morelos. On Psittacanthus calyculatus, 10 August 1926 Woronow 2604 [II, III] (PUR 48119).

Remarks. Uromyces euphlebius is morphologically close to $U$. socius having a similar urediniospore size and equivalent surface structure of teliospores. However, the teliospores of $U$. euphlebius are longer than those of $U$. socius.

Uromyces evastigatus Cumm., Mycologia 31. 173. 1939. (Figures 2 C, 5 E-F, 6 8-9).

Type. EL SAVADOR, SAN SALVADOR: $650-850 \mathrm{~m}$ a.s.1., 30 March 1922, 24 April 1922. On Phthirusa pyrifolia (Kunth) Eichler, P. C. Standley 23106 [I, III] (LECTOTYPE selected here, PUR 34937, ISOLLECTOTYPE [I] BPI 0004660).

Spermogonia unknown. Aecidia amphigenous, subepidermal, erumpent, pulverulent, grouped in areas of $1.5 \mathrm{~mm}$ diam., yellowish. Peridial cells rhomboidal, outer wall smooth, inner wall verrucose, lateral and distal wall ca. $2 \mu \mathrm{m}$ thick. Aecidiospores catenulate, oblong to ellipsoidal or globose, (30-) 37-43 (-47) × (23-) 28-36 (-40) $\mu \mathrm{m}$, minutely verrucose, hyaline, distal wall 5-12 $\mu \mathrm{m}$ thick. Uredinia not seen. Telia amphigenous, subepidermal, in small chocolate-brown spots, in 1-2 mm diam., groups, pulverulent, ruptured epidermis conspicuous. Teliospores 


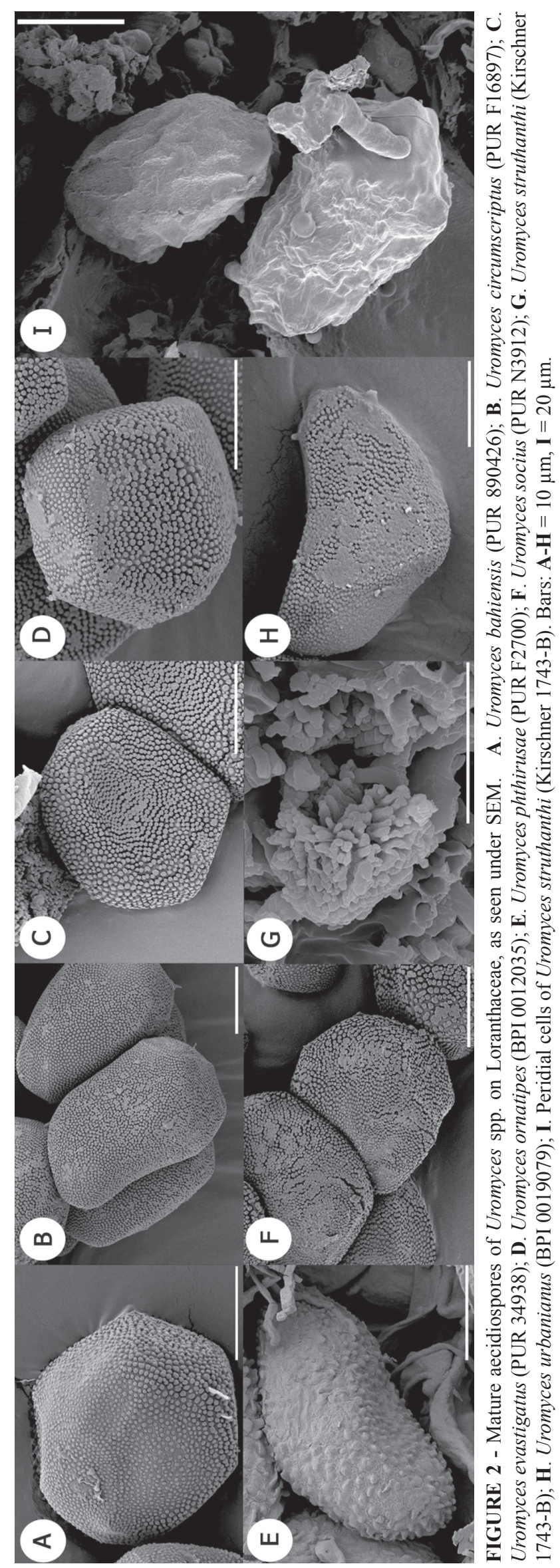




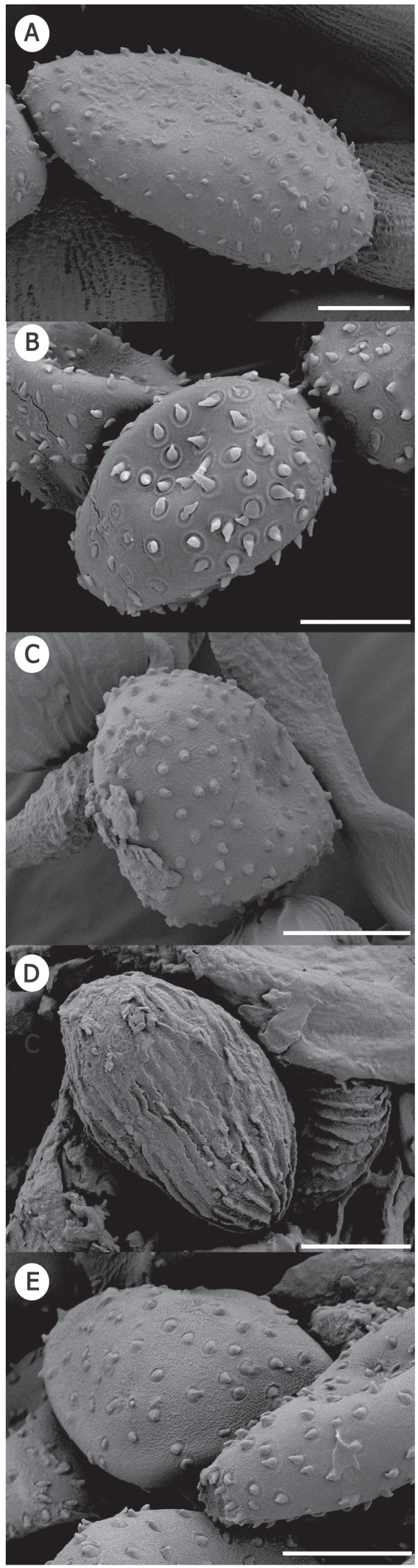

FIGURE 3 - Mature urediniospores of Uromyces spp. on Loranthaceae, as seen under SEM. A. Uromyces euphlebius (PUR 48119); B. Uromyces loranthi (type PUR F2697); C. Uromyces ornatipes (PUR 60498); D. Uromyces phthirusae (PUR F2700); E. Uromyces socius (PUR 60903). Bars $=10 \mu \mathrm{m}$. 1-celled, oblong-ellipsoidal, (40-) 41-48 (-52) $\times(16-) 20-$ $24(-25) \mu \mathrm{m}$, lateral wall 2-3 $\mu \mathrm{m}$ thick, distal wall 6-9 $\mu \mathrm{m}$ thick, chestnut-brown, reticulate, germ pores not observed. Pedicels persistent, cylindrical, smooth, thin-walled, hyaline, 10-47 × 6-7 $\mu \mathrm{m}$. Basidia and basidiospores not observed.

On Phthirusa pyrifolia (Kunth) Eichler (Cummins \& Stevenson, 1956).

Distribution. Known only from El Salvador (Cummins \& Stevenson, 1956).

Additional specimen examined. EL SALVADOR, SAN SALVADOR: Tonacatepeque. On Phthirusa pyrifolia 30-31 December 1921, P. C. Standley 19437 [I, III] (PUR 34938); ibid., [I] (BPI 0004659).

Remarks. Uromyces evastigatus is morphologically close to $U$. urbanianus having similar sized teliospore. However, the teliospores of $U$. evastigatus are reticulate and have longer pedicels.

Uromyces loranthi Jacks. \& Holw., Mycologia 19: 54. 1927. (Figures 3 B, 5 G-H, 6 10-12).

Type. BRAZIL, SABARÁ: Minas On Loranthus sp., 2 December 1921, E.W. D. \& M.M. Holway 1358 [II, III] (PUR F2697).

Spermogonia and aecidia not seen. Uredinia amphigenous, subepidermal, in groups, on small cinnamonbrown spots, $1.5-3 \mathrm{~mm}$ diam., pulverulent, ruptured epidermis conspicuous and persistent. Paraphyses 40-50 $\times$ 5-7 $\mu \mathrm{m}$, cylindrical, aseptate, smooth, persistent, thinwalled, hyaline to slightly yellowish. Urediniospores ellipsoidal or obovoid, (30-) 33-40 (-42) × (22-) 23-29 (-38) $\mu \mathrm{m}$, light cinnamon-brown, finely and sparsely echinulate, 1-1.5 $\mu \mathrm{m}$ thick, spines abundant, germ pores 4 equatorial. Pedicels persistent or deciduous, cylindrical, smooth, thin-walled, hyaline, 10-13 $\times 3-5 \mu \mathrm{m}$. Telia amphigenous, mostly abaxial, subepidermal, in small brown spots, $0.8-1$ $\mathrm{mm}$ diam., in 2-3 $\mathrm{mm}$ diam. groups, flattened to globoid, pulverulent, ruptured epidermis conspicuous. Teliospores 1-celled, oblong to ellipsoidal, (25-) 26-42 (-45) $\times(19-) 20$ $23(-24) \mu \mathrm{m}$, lateral wall $1.5-2 \mu \mathrm{m}$ thick, distal wall 6-8 $\mu \mathrm{m}$ thick, yellowish to hyaline, smooth to finely-densely verrucose, germ pores not observed. Pedicels persistent, cylindrical, smooth, thin-walled, hyaline, $8-10(-15) \times(3-)$ 4-5 $\mu \mathrm{m}$. Basidia and basidiospores not observed.

On Loranthus sp. (Hennen et al., 1982, 2005; Jackson, 1927; Mendes et al., 1998).

Distribution. Known only from Brazil (Hennen et al., 1982, 2005; Jackson, 1927; Mendes et al., 1998).

Remarks. Uromyces loranthi differs from $U$. euphlebius, $U$. ornatipes, and Uromyces socius by the ornamentation of the teliospores which are not arranged in lines. Additionally, $U$. loranthi differs from $U$. circumscriptus, $U$. evastigatus, and $U$. urbanianus by having smaller teliospores. The paraphyses of $U$. loranthi are reported, described and illustrated here for the first time. 


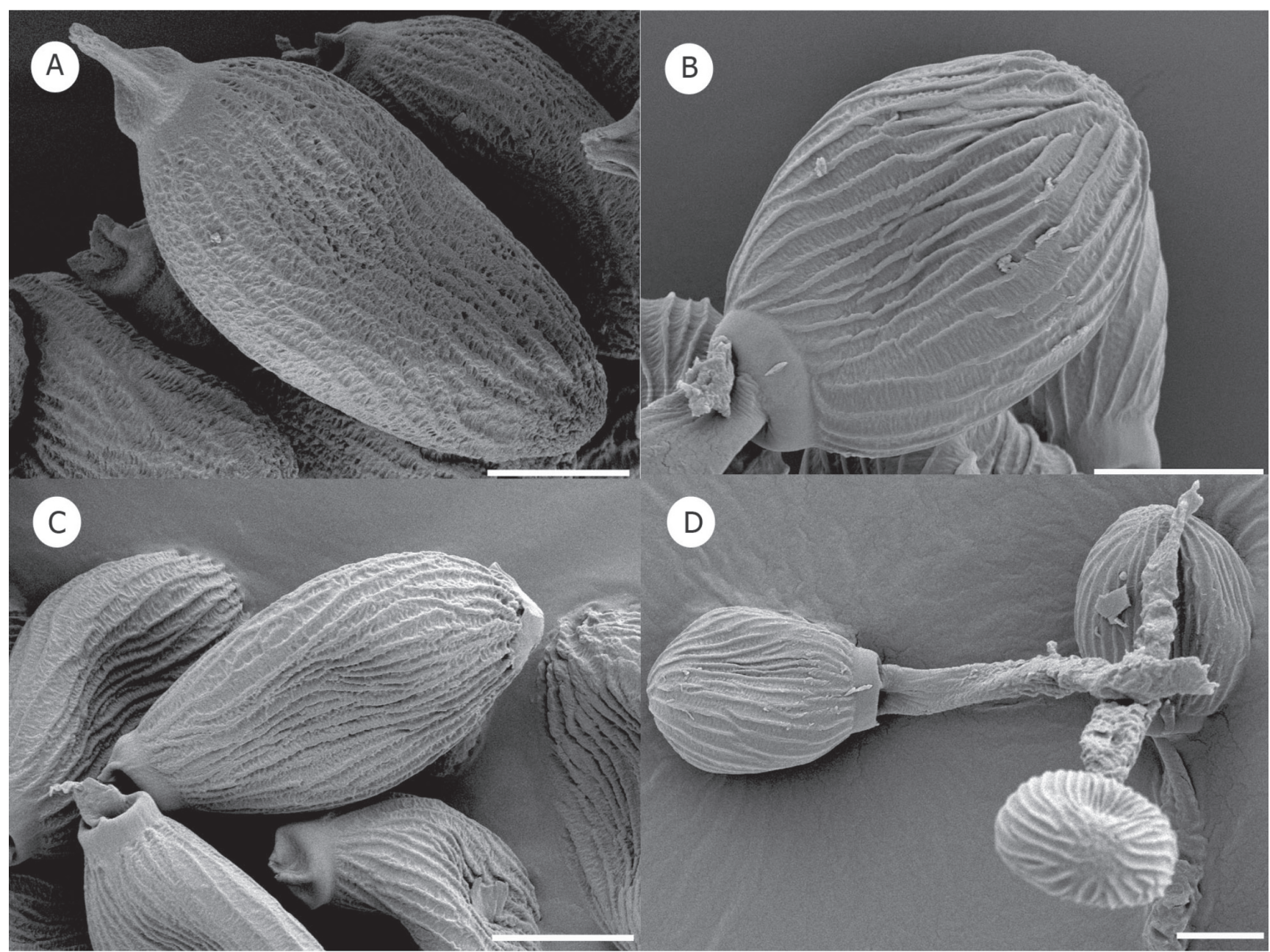

FIGURE 4 - Mature teliospores of Uromyces spp. on Loranthaceae, as seen under SEM. A. Uromyces euphlebius (type PUR 48119); B. Uromyces ornatipes (PUR 60498); C. Uromyces socius (PUR 13776); D. Uromyces ornatipes with details of pedicel (BPI 0012035). Bars $=10 \mu \mathrm{m}$.

Uromyces nilagiricus T. S. Ramakr. \& K. Ramakr., Proc. Indian Acad. Sci. Sect. B. 32 p. 104. 1950.

Type. INDIA, KOTAGIRI: On Loranthus sp., 25 April 1949, parasitic on Citrus reticulata, D. Murudarajan [I, III] n.v.

As no material is available, the following description is taken from Ramakrishnan \& Ramakrishnan (1950).

"Rust spot hypertrophied, up to $7 \mathrm{~mm}$ diam. Pycnidia amphigenous, subepidermal, oval, $240 \times 210 \mu \mathrm{m}$. Aecidia amphigenous, deeply sunk, cupulate, peridium of one layer of polygonal, colourless, thick-walled, verrucose cells. Aecidiospores angular, globose, catenulate, yellowish orange in colour, wall unevenly thickned, highly verrucose, $30 \times 25 \mu \mathrm{m}(24-33 \mu \mathrm{m} \times 18-30 \mu \mathrm{m})$. Uredinia wanting. Telia amphigenous, chocolate brown, suepidermal, erumpent. Teliospores 1-celled, elliptic to rhomboid, narrow towards the base, $36 \times 26 \mu \mathrm{m}(30-45 \times 21-30 \mu \mathrm{m})$ apex rounded, thickened up to $8 \mu \mathrm{m}$, orange brown in colour, wall smooth, pedicellate. Pedicels hyaline, up $130 \mu \mathrm{m}$ long."
On Loranthus sp. (Ramakrishnan \& Ramakrishnan, 1950).

Distribution. Only known from India (Ramakrishnan \& Ramakrishnan, 1950).

Remarks. According to Ramakrishnan \& Ramakrishnan (1950) the pycnidia and aecidia occur in swollen concavo-convex areas. The convexity may be oriented towards either surface. The aecidia are projected as short white columns with lacerated and recurved margins. The telia are not on the same spots as aecidia but occur on the same leaf. The wall of the teliospore is completely smooth as seen with the oil immersion objective.

According to Ramakrishnan \& Ramakrishnan (1950) $U$. nilagiricus is characterized by long teliospore pedicels and smooth teliospores. Thereby, it apparently differs from all other species of Uromyces on Loranthaceae. In order to confirm this species concept, it is necessary to locate and examine the type material or to designate a neotype. To date this is the only species of Uromyces on Loranthaceae known outside the Americas. 


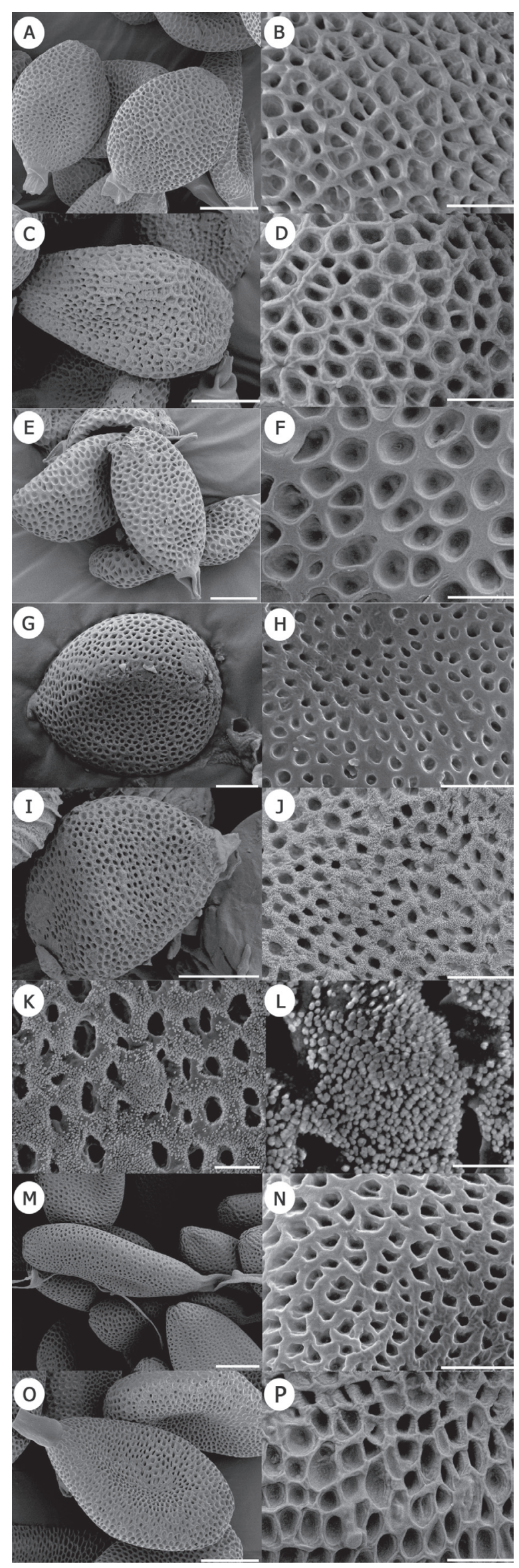

FIGURE 5 - Mature teliospores and ornamentation of Uromyces spp. on Loranthaceae, as seen by SEM. A, B. Uromyces bahiensis (PUR 890426) (Scale bar on the left hand side $=10 \mu \mathrm{m}$, on the right hand side $=3 \mu \mathrm{m}) ; \mathbf{C}, \mathbf{D}$. Uromyces circumscriptus; C. (PUR F11721) $($ bar $=10 \mu \mathrm{m}) ; \mathbf{D}$. (PUR N3910) $($ bar $=3 \mu \mathrm{m}) ; \mathbf{E}, \mathbf{F}$. Uromyces evastigatus (PUR 51731) (bar on the left $=10 \mu \mathrm{m}$; bar on the right $=3 \mu \mathrm{m}) ; \mathbf{G}, \mathbf{H}$. Uromyces loranthi (PUR F2697) (bar on the left $=10 \mu \mathrm{m}$, bar on the right $=3 \mu \mathrm{m}) ; \mathbf{I}, \mathbf{J}$. Uromyces phthirusae (PUR F2700) (bar on the left $=10 \mu \mathrm{m}$, bar on the right $=3 \mu \mathrm{m}) ; \mathbf{K}, \mathbf{L}$. Ornamentation of teliospores of Uromyces phthirusae (PUR F2700) (bar on the left $=1 \mu \mathrm{m}$, bar on the right $=400 \mathrm{~nm}) ; \mathbf{M}, \mathbf{N}$. Uromyces struthanthi (Kirschner 1743-B) (bar on the left $=10 \mu \mathrm{m}$, bar on the right $=3 \mu \mathrm{m}$ ); O. Uromyces urbanianus (PUR 52982) $(\mathrm{bar}=10 \mu \mathrm{m}) ;$ P. Uromyces urbanianus (PUR 64318) (bar $=3 \mu \mathrm{m})$. 

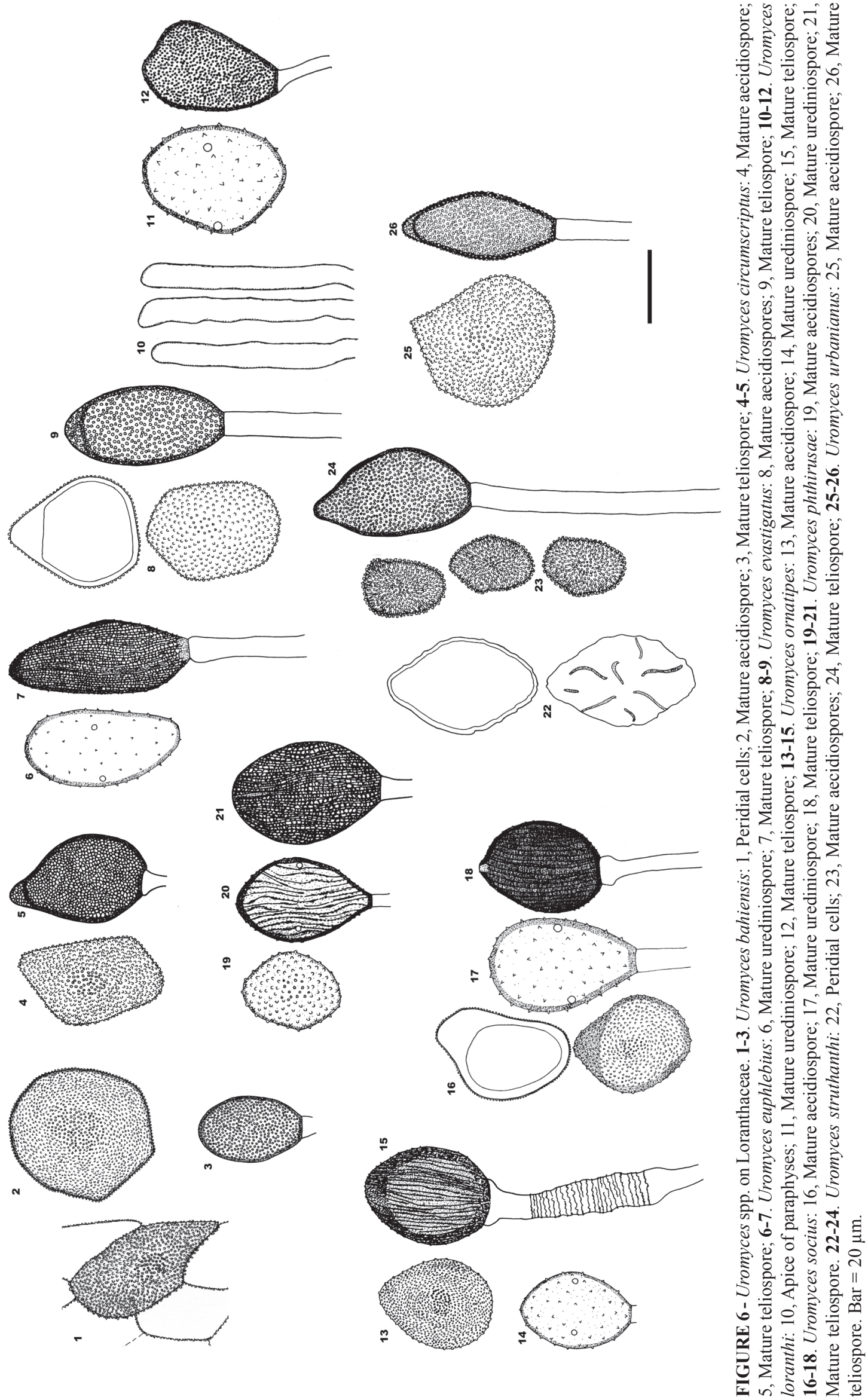
Uromyces ornatipes Arth., Bull. Torrey Club 42: 586. 1915. (Figures 2 D, 3 C, 4 B, D, 6 13-15).

Type. MEXICO: Cape San Lucas. On Phrygilanthus sonorae (S. Watson) Standl, March 1911, J.N. Rose 16396 [I, II, III] (LECTOTYPE selected here, PUR 13775; ISOLECTOTYPES [I] (BPI 0012034); [I, III] (BPI 0012035).

Spermogonia not seen. Aecidia amphigenous, 0.5-1 mm diam., subepidermal, erumpent, pulverulent, hyaline. Peridial cells (25-) 26-32 (-33) × (18-) 19-21 (-23) $\mu \mathrm{m}$, rhomboidal, overlapping, outer wall smooth, inner wall verrucose. Aecidiospores catenulate, angular, globoid or ellipsoidal, (24-) 26-32 (-35) × 22-26 (-28) $\mu \mathrm{m}$, verrucose, or very inconspicuously verrucose, hyaline. Uredinia few, amphigenous, subepidermal, on spots of 1.5-3 mm diam., brown, pulverulent, ruptured epidermis conspicuous. Urediniospores ellipsoidal, (25-) 26-32 (-33) × (20-) 21-24 (-25) $\mu \mathrm{m}$, wall golden-brown, echinulate, 1.5-2.5 $\mu \mathrm{m}$ thick, germ pores indistinct. Pedicels deciduous. Telia amphigenous, subepidermal, in small yellowish-brown spots, 0.3-0.6 mm diam., blackish to brown, subpulverulent, ruptured epidermis conspicuous. Teliospores 1-celled, ellipsoidal, (34-) 35$39(-40) \times(23-) 25-28(-29) \mu \mathrm{m}$, lateral wall $2-2.5 \mu \mathrm{m}$ thick, distal wall 5-7 $\mu \mathrm{m}$ thick, dark chocolate brown, often opaque, surface longitudinally rugose-striate, germ pores not observed. Pedicels persistent, cylindrical, smooth, thin-walled, hyaline, 60-70 × 7-9 $\mu \mathrm{m}$, wider $(10$ $14 \mu \mathrm{m}$ ) towards the base which is strongly transversely rugose. Basidia and basidiospores not observed.

On Phrygilanthus sonorae Standl. [= Loranthus sonorae (S. Watson) Standl.] (Arthur, 1915; Gallegos \& Cummins, 1981).

Distribution. Only known from Mexico (Arthur, 1915; Gallegos \& Cummins, 1981).

Additional specimen examined. MEXICO: Baja California. On Phrygilanthus sonorae, 14 March 1966, Lightle \& Gill 1-66 [I, II, III] (PUR 60498).

Remarks. Uromyces ornatipes differs from all other species of Uromyces on Loranthaceae by having pedicels which are smooth to strongly rugose. Furthermore, $U$. ornatipes is the only species with a longitudinally rugosestriate ornamentation of the teliospores.

Uromyces phthirusae Mayor ex Jackson, as "phtirusae", Mém. Soc. Neuchât. De Sc. Nat. 5: 448. 1913. (Figures 2 E, 3 D, 5 I-L, 6 19-21).

Uredo phthirusae (Mayor) Buriticá, in Buriticá \& Pardo-Cardona, Revta Acad. Colomb. Cienc. Exact. Fís. Nat. 20: 218. 1996. (Illegitimate name according to Berndt 2002)

Type. COLOMBIA, ANTIOQUIA DEPARTMENT: Cafetal de Camelia, Angelopolis. On Phthirusa pyrifolia $1800 \mathrm{~m}$ a.s.l., 24 August 1910, E. Mayor n.n. [I, II, III] (PUR F2700).
Spermogonia not seen. Aecidia amphigenous, 0.5-0.7 $\mathrm{mm}$ diam., subepidermal, erumpent, pulverulent, grouped in $1 \mathrm{~mm}$ diam., areas of, yellowish. Peridial cells not seen. Aecidiospores catenulate, angular, (28-) 29-30 (-32) $\times(21-)$ 22-25 (-26) $\mu \mathrm{m}$, verrucose, yellowish. Uredinia amphigenous, subepidermal, in groups on cinnamon-brown spots, $1.5-3 \mathrm{~mm}$ diam., pulverulent, ruptured epidermis conspicuous and persistent. Urediniospores ellipsoidal or obovoid, (30-) 33-40 (-42) × (18-) 20-24 (-27) $\mu \mathrm{m}$, wall light cinnamon-brown, longitudinally ridged to striate, 1-1.5 $\mu \mathrm{m}$ thick, with 4 equatorial germ pores. Pedicels deciduous. Telia amphigenous, subepidermal, on small yellowish-brown spots, $0.8-1 \mathrm{~mm}$ diam., grouped in areas of 1-3 mm diam., pale brown, pulverulent, ruptured epidermis conspicuous. Teliospores 1-celled, ellipsoidal to oblongellipsoidal, (35-) 38-46 (-53) × (17-) 20-25 (-26) $\mu \mathrm{m}$, lateral wall 3-4 $\mu \mathrm{m}$ thick, distal wall ca. $5 \mu \mathrm{m}$ thick, brown, surface reticulate, with very fine ornamentation and an apical germ pore. Pedicels persistent, cylindrical, smooth, thin-walled, hyaline, short, 8-10 $\times$ 4-5 $\mu \mathrm{m}$. Basidia and basidiospores not observed.

On Phthirusa pyrifolia (Kunth) Eichler (Chardon \& Toro, 1930; Dennis, 1970; Kern et al., 1933; Pardo Cardona, 1994).

Distribution. Known only in Colombia (Chardon \& Toro, 1930; Dennis, 1970; Kern et al., 1933; Pardo Cardona, 1994).

Additional specimen examined. COLOMBIA, ANTIOQUIA DEPARTMENT: Cafetal de Camelia, Angelopolis. On Phthirusa pyrifolia, $1800 \mathrm{~m}$ a.s.l., 24 August 1910, E. Mayor n.n. [III] (BPI 0013083); ibid., [II, III] (BPI 845455).

Remarks. Uromyces phthirusae differs from most species of Uromyces on Loranthaceae by having sparse verrucose aecidiospores and urediniospores which are longitudinally ridged to striate with four equatorial germ pores. Under SEM, a very fine reticulate ornamentation on the surface of teliospores of $U$. phthirusae was observed. This type of ornamentation is reported here for the first time for teliospores of a species of Uromyces. The aecidiospores of $U$. phthirusae are reported, described, and illustrated here for the first time.

Uromyces socius Arth. \& Holw., Am. J. Bot. 5: 437. 1918. (Figures 2 F, 3 E, 4 C, 6 16-18).

Type. GUATEMALA: Antigua. On Loranthus sp., 3 March 1916, Holway 545 [I, II, III] (PUR 13778).

Spermogonia not seen. Aecidia amphigenous, 0.5-0.9 mm diam., subepidermal, erumpent, pulverulent, grouped in of $1.5 \mathrm{~mm}$ diam. areas, yellowish. Peridial cells (35-) 39-46 (-50) × (16-) 18-25 (-30) $\mu \mathrm{m}$, rhomboidal, yellowish to hyaline, outer wall smooth, inner wall verrucose. Aecidiospores catenulate, angular, (30-) 32-36 $(-38) \times(16-)$ 25-31 (-33) $\mu \mathrm{m}$, verrucose, lateral wall 2.5-3 
$\mu \mathrm{m}$ thick, distal wall $c a$. $10 \mu \mathrm{m}$ thick, hyaline to yellowish. Uredinia amphigenous, subepidermal, in groups in small cinnamon-brown spots, $1.5-2 \mathrm{~mm}$ diam., pulverulent, ruptured epidermis conspicuous. Urediniospores ellipsoidal or obovoid, (40-) 41-52 (-56) × (19-) 22-26 (-27) $\mu \mathrm{m}$, wall light cinnamon-brown, echinulate, 1-2 $\mu \mathrm{m}$ thick, with 4 equatorial germ pores. Pedicels persistent, cylindrical, 30-45 × 7-8 $\mu \mathrm{m}$, smooth, thin-walled, hyaline. Telia amphigenous, subepidermal, in small yellowish-brown spots, $0.5-1.5 \mathrm{~mm}$ diam., in groups in $1-2 \mathrm{~mm}$ diam., areas, pale brown, pulverulent, ruptured epidermis conspicuous. Teliospores 1-celled, ellipsoidal to oblong-ellipsoidal, (32-) $34-42(-47) \times(20-) 21-25(-27) \mu \mathrm{m}$, lateral wall $3 \mu \mathrm{m}$ thick, distal wall $8-9 \mu \mathrm{m}$ thick, dark chestnut-brown, surface longitudinally striate, with distal germ pore. Pedicels persistent, cylindrical, smooth, thin-walled, hyaline, 10-45 $(-65) \times 4-8 \mu \mathrm{m}$. Basidia and basidiospores not observed.

On Struthanthus densiflorus (Benth.) Standl., S. haenkeanus Standl, S. palmeri Kuijt, S. pyrifolius Blume, S. sp., Loranthus crassipes Oliv., and L. sp. (Arthur, 1918; Carrion \& Galvan, 1987; Gallegos \& Cummins, 1981).

Distribution. Known from El Salvador, Guatemala and Mexico (Arthur, 1918; Carrion \& Galvan, 1987; Gallegos \& Cummins, 1981).

Additional specimens examined. EL SALVADOR, SAN SALVADOR: Santa Tecla, Experimental Station. On Struthanthus sp., 4 November 1945, F. L. Wellman 899 [II, III] (PUR 51732); ibid., F. L. Wellman 900 [II, III] (PUR 517733); MEXICO, SONORA: Guaymas. On Struthanthus haenkianus, 22 October 1965, J.F. Hennen 65-285 [I, III] (PUR 60903); MEXICO, SONORA: Sierra Madre, El Río Bonito about La Nopalera, 10 May 1939, C. H. Muller 3629 [III] (BPI 0016659); MEXICO, SONORA: Alamos. On Struthanthus palmeri, 2 July 1992, T. R. Van Devender \& S. L. Friedman 92-683 [I] (PUR N3912); MEXICO, PUEBLA: On Loranthus sp., C. A. Purpus [I] (BPI 0016648); GUATEMALA: Huehuetenango. On Struthanthus densiflorus, 22 January 1917, Holway 765 [II, III] (PUR 17381), ibid., [III] (BPI 006651, 0016658); Antigua. On Loranthus sp.1 March 1916, Holway 539 [I, II, III] (PUR 13780), ibid., [I] (BPI 0016650), ibid., [II] (BPI 0016641); Panajachel, Solola, 3 January 1917, Holway 665 [II, III] (PUR 13779), ibid., [III] (BPI 0016649); Solola. On Loranthus crassipes, 1 February 1915, Holway 169 [I, III] (PUR 13777), ibid., [III] (BPI 006651); San Lucas Toliman, 3 February 1915, Holway 185 [I, III] (PUR 13776), ibid., [III] (BPI 006652, 0016653); GUATEMALA, ANTIGUA: Sacatépequez. On Struthantus sp., 1 March 1916, E. W. D. Holway 539 [III] (BPI 0016656); EL SALVADOR, LA CEIBA: On Struthantus pyrifolius, 10 December 1945, F.L. Wellman 1143 [II] (BPI 0016660).

Remarks. Uromyces socius is morphologically close to $U$. euphlebius by having similar sized urediniospore and similarly ornamented teliospores. However, the teliospores of $U$. euphlebius are longer in contrast to those of $U$. socius.
Uromyces struthanthi Perd.-Sánch., sp. nov. (Figures 2 G, I 5 M-N, 6 22-24).

Type. PANAMA, CHIRIQUÍ PROVINCE: Boquete, Finca Arco Iris. on leaves of Struthanthus sp. (Loranthaceae), 1,300 m a.s.1., 7 March 2003, R. Kirschner 1743 B [I, III] (holotype PMA, ISOTYPE M-0141257).

Etymology. Named after the host genus, Struthanthus (Loranthaceae).

MycoBank \# MB805147.

Spermogonia not seen. Aecidia hypophyllous, 0.5$1.5 \mathrm{~mm}$ diam., subepidermal, erumpent, not pulverulent, yellowish. Peridial cells (40-) 42-45 (-48) × (27-) 29$30(-32) \mu \mathrm{m}$, oblong, smooth to roughened, hyaline to yellowish. Aecidiospores (15-) 17-20 (-25) × (8-) 10$13(-15) \mu \mathrm{m}$, catenulate, angular, spinose-echinulate, yellowish to hyaline. Uredinia not seen. Telia hypophyllous, subepidermal, in small yellowish-brown spots, $0.8-1 \mathrm{~mm}$ diam., grouped in 2-3 mm diam. areas, flattened to globoid, pulverulent, ruptured epidermis conspicuous. Teliospores 1-celled, oblong-ellipsoidal, (35-) 42-47 (-50) $\times(20-) 23-$ $26(-27) \mu \mathrm{m}$, lateral wall $2 \mu \mathrm{m}$ thick, distal wall 8-9 $\mu \mathrm{m}$ thick, yellowish-brown, reticulate-foveate, germ pores not observed. Pedicels persistent, cylindrical, 8-80 (-90) $\times$ 4-5 $\mu \mathrm{m}$, smooth, thin-walled, hyaline. Basidia and basidiospores not observed.

\section{On Struthanthus sp.}

Distribution. Known only from Panama.

Remarks. No spermogonia, uredinia, basidia or basidiospores were found in this collection. Telia are abundant. The new species is characterized by having large teliospore pedicels and spinose-echinulate aecidiospores that differentiates it from all other species of Uromyces on Loranthaceae.

Uromyces urbanianus P. Henn., Hedwigia 36: 213. 1897. (Figures 2 H, 5 O-P, 6 25-26).

Type. TRINIDAD, ST. ANNS: On Oryctanthus spicatus, August 1896, J. H. Hart n.n. [III] (PUR F155572).

Spermogonia not seen. Aecidia hypophyllous, 0.5-1 $\mathrm{mm}$ diam., subepidermal, erumpent, pulverulent, grouped in areas of 1-3 mm diam., yellowish. Peridial cells (37-) 38-54 (-72) $\times(22-) 28-39(-40) \mu \mathrm{m}$, rhomboidal, outer wall smooth, inner wall verrucose, hyaline or slightly yellowish. Aecidiospores (40-) 42-50 × (20-) 29-40 (-42) $\mu \mathrm{m}$, catenulate, angular to globose, verrucose, hyaline. Uredinia not seen. Telia amphigenous, mostly abaxial, subepidermal, in small brown spots, $0.8-1 \mathrm{~mm}$ diam., grouped in $2 \mathrm{~mm}$ diam.areas, flattened to globoid, pulverulent, ruptured epidermis conspicuous. Teliospores 1-celled, oblong to ellipsoidal, (35-) 39-45 (-48) × (20-) 21-24 (-25) $\mu \mathrm{m}$, lateral wall 2-4 $\mu \mathrm{m}$ thick, distal wall 5-7 $\mu \mathrm{m}$ thick, yellowishbrown, surface finely-closely verrucose-striate, germ pores not observed. Pedicels persistent, cylindrical, smooth, 
thin-walled, hyaline, $8-25(-30) \times 5-7 \mu \mathrm{m}$. Basidia and basidiospores not observed.

On Phoradendron sp., Phrygilanthus acutifolius (Ruiz \& Pav.) Eichler, Psittacanthus calyculatus (DC.) G. Don, Psittacanthus americanus (L.) Mart., Psittacanthus sp., Oryctanthus spicatus Eichler and Struthanthus complexus Eichler. (Buriticá \& Pardo Cardona, 1996; Chardon \& Toro, 1930; Dennis, 1970; Gallegos \& Cummins, 1981; Hennen et al., 1982, 2005; Kern et al., 1933; Lindquist, 1982; Mendes et al., 1998; Pardo Cardona, 1994, 1998; Salazar-Yepes \& Buriticá, 2002; Salazar-Yepes et al., 2002).

Distribution. Known from Argentina, Colombia, Guatemala, Honduras, Mexico, and Trinidad (Buriticá \& Pardo Cardona, 1996; Chardon \& Toro, 1930; Dennis, 1970; Gallegos \& Cummins, 1981; Hennen et al., 1982, 2005; Kern et al., 1933; Lindquist, 1982; Mendes et al., 1998; Pardo Cardona, 1994, 1998; Salazar-Yepes \& Buriticá 2002; Salazar-Yepes et al., 2002).

Additional specimens examined. ARGENTINA, SALTA: Pampa Grande. On Phrygilanthus acutifolius, [III] (PUR F16916); MEXICO: Lepic. On Psittacanthus americanus, 18 November 1971, Cummins 71-491 [III] (PUR 64318); Nayarit. On Psittacanthus sp., 14 December 1970, Cummins 70-271 [III] (PUR 63711); GUATEMALA, BARCENA: On Psittacanthus sp., April 1942, Müller 112 [I, III] (PUR 51132); BRAZIL, RÍO DE JANEIRO: On Struthanthus complexus January 1896, Ule 2123 [III] (PUR F2699); COLOMBIA, EL VALLE: Meléndes, Hacieda Las Palmas. On Phoradendron sp., I April 1938, C. Garces 48 [III] (PUR F 9483); HONDURAS: Escuela Agrícola Panamericana. On Psittacanthus calyculatus, 12 January 1951, Müller 444 [III] (PUR 52982).

Remarks. Uromyces urbanianus differs from $U$. circumscriptus byhaving verrucose-striateteliosporesinstead of reticulate-striate teliospores as in $U$. circumscriptus. In addition to this, the teliospores of $U$. urbanianus are longer than those of $U$. circumscriptus. Uromyces urbanianus is morphologically close to U. evastigatus because of similarly sized teliospores. However, teliospores in U. urbanianus are finely and densely verrucose instead of reticulate as in $U$. evastigatus and teliospore pedicels are smaller in $U$. urbanianus.

\section{Key to species of Uromyces on Loranthaceae}

Surface of teliospores longitudinally striate or rugose striate

Pedicels of teliospores with lower part strongly transversely rugose

Teliospores 35-39 $\mu \mathrm{m}$ long............Uromyces ornatipes Pedicels of teliospores completely smooth

Teliospores 47-50 $\mu \mathrm{m}$ long................Uromyces euphlebius Teliospores 34-42 $\mu \mathrm{m}$ long...................Uromyces socius Surface of teliospores smooth, smooth to finely-densely verrucose, reticulate, finely and densely verrucose, or reticulate-striate. Species with uredinia
Urediniospores echinulate Uromyces loranthi Urediniospores longitudinally ridged to striate...Uromyces phthirusae

Species without known uredinia

Teliospores mostly less than $40 \mu \mathrm{m}$ long

Teliospores ellipsoidal, reticulate-striate, 36-41 x 19-23 $\mu \mathrm{m}$....Uromyces circumscriptus

Teliospores oblong-ellipsoidal, reticulate, 25-29 x 16-18 $\mu \mathrm{m}$......Uromyces bahiensis

Teliospores mostly more than $40 \mu \mathrm{m}$ long

Teliospores verrucose-striate, $39-45 \times 21-24 \mu \mathrm{m}$.

Uromyces urbanianus

Teliospores reticulate, 41-48 x 20-24 $\mu \mathrm{m}$..........Uromyces evastigatus

Teliospores reticulate-foveate, $42-47 \times 23-26 \mu \mathrm{m}$.

Uromyces struthanthi

Teliospores smooth, 30-45 x 21-30 $\mu \mathrm{m}$. Uromyces nilagiricus

\section{ACKNOWLEDGEMENTS}

We are grateful to R. Kirschner for providing the specimen from Panama and for critically reading the manuscript, M. Ruppel for technical work of SEM, J. R. Hernández for providing literature, and the Herbaria BPI and PUR for loan of material. The present study was supported by the DAAD (German Academic Exchange Service), DFG (German Research Society), the UNACHI (Universidad Autónoma de Chiriquí), the ANAM (Autoridad Nacional del Ambiente, Panama), and the LOEWE excellence initiative of the state of Hesse within the framework of the Cluster for Integrative Fungal Research (IPF).

\section{REFERENCES}

Arthur JC (1915) New species of Uredineae IX. Bulletin of Torrey Botanical Club 42:585-593.

Arthur JC (1918) Uredinales of Guatemala based on collections by E.W. D. Holway. II. Aecidiaceae, exclusive of Puccinia and form genera. American Journal of Botany 5:420-446.

Berndt R (2002) New species, reports and observations on rust fungi. Nova Hedwigia 75:415-431.

Buriticá P, Pardo Cardona VM (1996) Flora Uredineana Colombiana. Revista Academia Colombiana de Ciencias 20:183236.

Carrion G, Galvan M (1987) Plant pathogenic fungi from the state of Veracruz. Uredinales V. Revista Mexicana de Micología 3:149159.

Chardon C, Toro RA (1930) Mycological explorations of Colombia. Journal of the Department of Agriculture of Puerto Rico 14:195-369.

Cummins GB, Stevenson JA (1956) A check list of North American rust fungi (Uredinales). Plant Disease Reporter 240(Suppl.):109192. 
Cummins GB, Hiratsuka Y (2003) Illustrated genera of rust fungi. $3^{\text {rd }}$ Ed. Minnesota, MN, USA. APS Press.

Dennis RWG (1970) Fungus flora of Venezuela and adjacent countries. Kew Bulletin Additional Series III.

Gallegos ML, Cummins GB (1981) Uredinales (Royas) de Mexico. Vol. 2. Culiacan, Mexico. Instituto Nacional de Investigaciones Agrícolas.

Hennen JF, Hennen MM, Figueiredo MB (1982) Index of the rust fungi (Uredinales) of Brazil. Arquivos do Instituto Biologico 49 (Suppl):1-201.

Hennen JF, Figueiredo MB, de Carvalho Jr. AA, Hennen PG (2005) Catalogue of the species of plant rust fungi (Uredinales) of Brazil. Rio de Janeiro, RJ, Brazil. Instituto de Pesquisas, Jardim Botânico do Rio de Janeiro.

Jackson HS (1927) The rusts of South America based on the Holway collections - II. Mycologia 19:51-65.

Kern FD, Thurston Jr. HW, Whetzel HH (1933) Annotated index of the rusts of Colombia. Mycologia 25:448-503.

Kuiji J (1978) Commentary on the mistletoes of Panama. Annals of the Missouri Botanical Garden 65:736-763.

Lindquist JC (1982) Royas de la República Argentina y Zonas Limítrofes. Córdoba, Argentina. Instituto Nacional de Tecnología Agropecuaria.

Mendes MAS, da Silva VK, Dianese JC, Ferreita, MASU, Santos CEN, Neto EG, Urben AF, Castro C (1998) Fungos em plants no Brasil. Brasília, DF. Embrapa SPI.

Mujica F, Oehrens BE (1967) Segunda addenda a flora fungosa Chilena. Santiago, Chile. Universidad de Chile. Boletín Técnico 27.
Mujica F, Vergara C (1945) Flora Fungosa Chilena. Índice Preliminar de los Huéspedes de los Hongos Chilenos y sus Referencias Bibliográficas. Santiago, Chile. Imprenta Stanley.

Pardo Cardona VM (1994) Índice comentado de las royas (Fungi, Uredinales) del departamento de Antioquia, Colombia. Revista del Instituto Colombiano de Ciencias Naturales y Ecología 5:99-172.

Pardo Cardona VM (1998) Distribución de las especies colombianas de Uredinales según los grupos taxonómicos de sus hospederos. Revista Facultad Nacional de Agronomía de Medellin 51:285-319.

Ramakrishnan TS, Ramakrishnan K (1950) Additions to fungi of Madras VIII. Proceedings of the Indian Academy of Science, Section B 32:102-110.

Salazar-Yepes M, Buriticá P (2002) Nuevos registros de hospedantes para la uredomicota colombiana. Revista Facultad Nacional de Agronomía de Medellín 55:1615-1632.

Salazar-Yepes M, Buriticá P, Cadena-Gómez G (2002) Implicaciones de los estudios sobre biodiversidad de los Uredinales (Royas) en la región cafetera colombiana. Cenicafe 53:219-238.

Shivas RG, Hyde KD (1997) Biodiversity of plant pathogenic fungi in the neotropics. In: Hyde KD (Ed.) Biodiversity of Tropical Microfungi. Hong Kong, China. Hong Kong University Press. pp 47-56.

Van der Merwe M, Ericson L, Walker J, Thrall PH, Burdon J (2007) Evolutionary relationships among species of Puccinia and Uromyces (Pucciniaceae, Uredinales) inferred from partial protein coding gene phylogenies. Mycological Research 3:163-175.

Vidal-Russel R, Nickrent DL (2008) Evolutionary relationships in the showy mistletoe family (Loranthaceae). American Journal of Botany 95:1015-1029. 